\title{
Efectos del entrenamiento con Xbox Kinect sobre la movilidad funcional en adultos mayores. Una revisión breve
}

\author{
Effects of training with Xbox Kinect on functional mobility in older adults. \\ A brief review \\ *Jordan Hernández Martínez, **María Rauch Gajardo, **Diego Rivas Coñapi, \\ **Paola Asenjo Flores, ***Catalina Asenjo Paredes, ***Monserrat Solis Millaguin.
}

Hernández, J.; Rauch, M.; Rivas, D.; Asenjo, P.; Asenjo, C. \& Solis, M. (2018). Efectos del entrenamiento con Xbox Kinect sobre la movilidad funcional en adultos mayores. Una revisión breve. Revista Ciencias de la Actividad Física UCM, $\mathrm{N}^{\circ}$ 19(2) julio-diciembre, 1-9. DOI: http://doi.org/10.29035/rcaf.19.2.2

\section{RESUMEN}

Objetivo: analizar los efectos del entrenamiento mediante consola Xbox Kinect sobre la movilidad funcional en adultos mayores. Método: se realizó una búsqueda bibliográfica sistemática en PUBMED. Se seleccionaron estudios cuantitativos clínicos experimentales en inglés realizados en los últimos seis años. Resultados: nueve estudios fueron detectados. Entre los principales hallazgos, el entrenamiento con consola Xbox Kinect incrementó la fuerza muscular, mejora del equilibrio, movilidad funcional, función cognitiva, propiocepción de rodilla y calidad de vida en adultos mayores. Conclusión: el entrenamiento con consola Xbox Kinect en adultos mayores mejora la movilidad funcional en este grupo etario.

\section{PALABRAS CLAVE}

Equilibrio, propiocepción, fuerza, extremidades inferiores.

\begin{abstract}
Objective: to analyze the effects of Xbox Kinect console training on functional mobility in older adults. Method: a systematic bibliographic search was performed in PUBMED. Experimental quantitative clinical studies in English were conducted over the last six years. Results: nine studies were detected. Among the main findings, training with the Xbox Kinect console increased muscle strength, improved balance, functional mobility, cognitive function, knee proprioception and quality of life in older adults. Conclusion: training with the Xbox Kinect console in older adults improved functional mobility in this age group.
\end{abstract}

Key words

Balance, proprioception, strength, lower extremities.

* Universidad de los Lagos de la carrera Técnico Deportivo Universitario, Integrante núcleo de investigación en salud, actividad física y deporte de la Universidad de los Lagos.

** Universidad Santo Tomás, Facultad de Salud, Escuela de Terapia Ocupacional, Osorno.

*** Estudiantes de la carrera Técnico Deportivo Universitario de la Universidad de los Lagos. 


\section{INTRODUCCIÓN}

En la actualidad la población de adultos mayores ha sufrido una incremento considerable (OMS, 2018), aumentando la esperanza de vida en este grupo etario (OMS, 2018), en Chile el promedio de vida es de 78.4 años (SENAMA, 2011). Este envejecimiento es un proceso natural, el cual trae cambios a nivel morfológico y fisiológico (Gómez, Rodríguez, Maldonado, Casajús, \& Ara, 2012). Dentro de estos cambios se puede evidenciar un descenso en el funcionamiento de los sistemas musculoesqueléticos y neurológicos (Relph \& Herrington, 2016), siendo relevante en la evidencia, la deficiencia en el sistema propioceptivo, el cual se encuentra relacionado a la capacidad y desempeño asociado al control motor (Ko, et al., 2017), pudiendo afectar la movilidad funcional en actividades de la vida diaria en esta población, una movilidad escasa se asocia a un adulto mayor más frágil y por ende con un mayor riesgo de sufrir una caída (Petronila, Aragón, \& Calvo, 2017) esto puede desencadenar lesiones graves como fracturas óseas en algunos casos, incapacidad a largo plazo, asociándose a una baja densidad ósea, que afecta la calidad de vida, siendo el adulto mayor más dependiente funcionalmente, lo que conduce a un mayor riesgo de morbilidad y mortalidad (Masud \& Morris, 2001). En Chile según la Encuesta de caracterización socioeconómica nacional (CASEN, 2015), un $85.5 \%$ de los adultos mayores son independientes funcionalmente, mientras que un $14.4 \%$ presentan dependencia funcional en sus actividades de la vida diaria. Por esto, es importante entregar tratamientos a los adultos mayores que ayuden a prevenir una vida de dependencia funcional. Trastornos en la fuerza, equilibrio y coordinación conducen a una vida de dependencia con un mayor riesgo de caídas en los adultos mayores, por tanto, es importante realizar intervenciones que ayuden a reducir estas alteraciones, siendo el ejercicio físico una buena opción, ya que permite disminuir la incidencia de caídas (Nelson et al., 2007) ejercicios de fuerza resistencia (Lima et al., 2018), potencia (Lopes, Pereira, Lodovico, Bento, \& Rodacki, 2016), resistencia aeróbica (Casas \& Izquierdo, 2012) o mediante vibraciones de cuerpo completo (Hernández \& Ramírez, 2017). Es importante la aplicación de terapia física para el tratamiento de trastornos sensoriales (en particular trastornos visuales y vestibulares), así como tareas motoras específicas para mejorar diferentes aspectos del equilibrio, la fuerza, la flexibilidad, la integración sensorial, tareas que requieren atención (Shimada et al. 2004; Silsupadol et al., 2006).

En un estudio realizado por Burke et al., 2009 afrontan el diseño de juegos basados en computadora y su efecto en la recuperación funcional. Intervenciones en realidad virtual, han sido una opción a las terapias convencionales en adultos mayores encontrando consolas PlayStation Move ${ }^{\circledR}$ de Sony ${ }^{\circledR}$, Xbox Kinect ${ }^{-}$de Microsoft ${ }^{\circledR}$, o Wii ${ }^{\circledR}$ de Nintendo ${ }^{\circledR}$, (Smith \& Schoene, 2012). Xbox Kinect ${ }^{\circledR}$ ha demostrado ser una herramienta interesante para evaluar la función física, utilizada en poblaciones clínicas examinando aspectos en la función como marcha y equilibrio (Galna et al., 2014; Vernon et al., 2015).

Las intervenciones mediante video juegos han mostrado una mayor adherencia en las terapias y mejoras en parámetros de funcionalidad como el equilibrio, movilidad y calidad de vida en la población geriátrica (Staiano \& Calvert, 2011a). En un estudio realizado por (Padala et al., 2017) se observaron mejoras en la escala de balance Berg, en una intervención de ocho semanas con tres sesiones a la semana de cuarenta y cinco minutos con consola Nintendo Wii-Fit. Se han observado mejoras en la fuerza de miembros inferiores y movilidad funcional en adultos mayores sometidos a un entrenamiento con consola Wii Fit durante 10 semanas teniendo 2 sesiones por semana de 35 minutos (Jorgensen, Laessoe, Hendriksen, Nielsen, \& Aagaard, 2013). A diferencia de otras consolas Kinect para Xbox, no restringe a los pacientes al mismo lugar, y un paciente puede avanzar o moverse para controlar el juego. Involucra el movimiento de todo el cuerpo, que es similar al del mundo real, sin el uso de un controlador o parado sobre un tablero angosto lo cual puede entregar una mayor amplitud de movimiento en el espacio (Sin \& Lee., 2013). El presente estudio busca observar mediante una revisión actualizada los efectos del entrenamiento mediante Xbox Kinect sobre la movilidad funcional en adultos mayores. 
Tabla 1

Características experimentales de los estudios seleccionados.

\begin{tabular}{|c|c|c|c|c|}
\hline Autores & Sujetos & Diseño de estudio e intervención & $\begin{array}{l}\text { Duración de } \\
\text { intervención }\end{array}$ & $\begin{array}{l}\text { Principales } \\
\text { resultados }\end{array}$ \\
\hline Bierla, 2015 & $\begin{array}{l}13 \text { adultos mayores } \\
\text { ( } 3 \text { hombres, } 10 \text { mujeres) } \\
\text { GC } n=7 \\
\text { (edad, } 82.6 \pm 6.9 \text { años), } \\
\text { GE } n=6 \text { (edad, } 82 \pm 2.4 \text { años). }\end{array}$ & $\begin{array}{l}\text { GE Xbox } 360 \text { kinect programa de } \\
\text { ejercicio: Your Shape; Fitness Evolved } \\
\text { y adventure Kinect. GC siguió con su } \\
\text { vida diaria como de costumbre. }\end{array}$ & $\begin{array}{l}30 \min \times 3 \\
\text { semanas } \times 3 \\
\text { sesiones }\end{array}$ & $\begin{array}{l}\uparrow \mathrm{E} \\
\uparrow \mathrm{MF}\end{array}$ \\
\hline Shih et al., 2016 & $\begin{array}{l}10 \text { adultos mayores } \\
\text { ( } 7 \text { hombres, } 3 \text { mujeres) } \\
\text { GC (edad, } 68.8 \pm 9.67 \text { años) } \\
10 \text { Adultos mayores } \\
\text { ( } 9 \text { hombres, } 1 \text { mujer) } \\
\text { GE (edad, } 67.5 \pm 9.96 \text { años). }\end{array}$ & $\begin{array}{l}\text { GE Xbox } 360 \text { kinect + ejercico de } \\
\text { equilibrio programa de ejercicio: } \\
\text { Reaching task } 1 \text {, Reaching task } 2 \text {, } \\
\text { Obstacle avoidance, Marching. GC } \\
\text { programa de entrenamiento con } \\
\text { ejercicios de equilibrio. }\end{array}$ & $\begin{array}{l}30 \min \times 8 \\
\text { semanas } \times 2 \\
\text { sesiones }\end{array}$ & $\begin{array}{l}\uparrow \mathrm{MF} \\
\uparrow \mathrm{E} \\
\uparrow \mathrm{EP}\end{array}$ \\
\hline Grigorova et al., 2015 & $\begin{array}{l}10 \text { adultos mayores } \\
8 \text { hombres, } 2 \text { mujeres } \\
\text { (edad, } 80.6 \pm 7.25 \text { años) }\end{array}$ & $\begin{array}{l}\text { Xbox } 360 \text { Kinect programa de } \\
\text { ejercicio: Skiing, Boxing, Shootout } \\
\text { and Dances }\end{array}$ & $\begin{array}{l}20 \text { a } 30 \min \times 4 \\
\text { semanas } \times 5 \\
\text { sesiones }\end{array}$ & $\begin{array}{l}\uparrow \mathrm{E} \\
\uparrow \mathrm{FC}\end{array}$ \\
\hline Lee, 2013 & $\begin{array}{l}14 \text { adultos mayores grupo; } \\
\text { GE n=7; GC n=7 }\end{array}$ & $\begin{array}{l}\text { GE Xbox } 360 \text { Kinect terapia } \\
\text { ocupacional convencional+programa } \\
\text { de ejercicio: Kinect sports } \\
\text { (Boxing and Bowling) and Kinect } \\
\text { adventure. GC terapia ocupacional } \\
\text { convencional. }\end{array}$ & $\begin{array}{l}60 \min \times 6 \\
\text { semanas } \times 3 \\
\text { sesiones }\end{array}$ & $\begin{array}{l}\uparrow A V D \\
\uparrow F M\end{array}$ \\
\hline Lee et al., 2017 & $\begin{array}{l}47 \text { adultos entre } \\
\text { (edad, } 20 \text { y } 75 \text { años) } \\
\text { GE } n=26 ; \text { GC } n=21\end{array}$ & $\begin{array}{l}\text { GE tratamiento estándar (ejercicios } \\
\text { de equilibrio) +programa de ejercicio } \\
\text { con Xbox } 360 \text { Kinect: Kinect Sports, } \\
\text { Kinect Adventures. GC tratamiento } \\
\text { estándar (ejercicios de equilibrio). }\end{array}$ & $\begin{array}{l}90 \min \times 6 \\
\text { semanas } \times 2 \\
\text { sesiones }\end{array}$ & $\uparrow E$ \\
\hline Kim et al., 2013 & $\begin{array}{l}32 \text { adultos mayores } \\
\text { GE } n=18 \\
\text { (edad, } 68.3 \pm 3.7 \text { años) } \\
\text { GC } n=14 \\
\text { (edad, } 66.2 \pm 3.9 \text { años) }\end{array}$ & $\begin{array}{l}\text { GE Xbox } 360 \text { Kinect programa de } \\
\text { ejercicio: Your Shape Fitness. GC } \\
\text { siguió con su vida diaria como de } \\
\text { costumbre. }\end{array}$ & $\begin{array}{l}60 \min \times 8 \\
\text { semanas } \times 3 \\
\text { sesiones }\end{array}$ & $\uparrow F M$ \\
\hline Sato et al., 2015 & $\begin{array}{l}54 \text { adultos mayores } \\
\text { GE } n=28 \\
\text { (edad, } 70.1 \pm 5.4 \text { años) } \\
\text { GC } n=26 \\
\text { (edad, } 68.5 \pm 5.5 \text { años) }\end{array}$ & $\begin{array}{l}\text { GE Xbox } 360 \text { Kinect programa de } \\
\text { ejercicio: apple game, tightrope } \\
\text { standing game, ballon popping } \\
\text { game, y One-leg standing game. GC } \\
\text { siguió con su vida diaria como de } \\
\text { costumbre, }\end{array}$ & $\begin{array}{l}40-60 \text { min } \times 2- \\
3 \text { por semana } \\
\text { (total } 24 \\
\text { sesiones) }\end{array}$ & $\begin{array}{l}\uparrow F M \\
\uparrow E\end{array}$ \\
\hline Sadeghi et al., 2017 & $\begin{array}{l}30 \text { adultos mayores } \\
\text { (edad, } 73 \pm 7 \text { años) } \\
\text { GE } n=15 ; \text { GC } n=15\end{array}$ & $\begin{array}{l}\text { GE Xbox } 360 \text { Kinect programa de } \\
\text { ejercicio: Kinect Sports. GC no } \\
\text { realizó intervención alguna. }\end{array}$ & $\begin{array}{l}40 \min \times 8 \\
\text { semanas } \times 3 \\
\text { sesiones }\end{array}$ & $\uparrow \mathrm{PAR}$ \\
\hline Karahan et al., 2015 & $\begin{array}{l}90 \text { adultos mayores } \\
\text { GC } n=42 ; \\
18 \text { mujeres, } 24 \text { hombres } \\
\text { (edad, } 71.5 \pm 4.7 \text { años) } \\
\text { GE n=48; } \\
21 \text { mujeres, } 27 \text { hombres } \\
\text { (edad, } 71.3 \pm 6.1 \text { años) }\end{array}$ & $\begin{array}{l}\text { GE Xbox } 360 \text { Kinect programa de } \\
\text { ejercicio: Kinect Adventures, Kinect } \\
\text { Sports. GC ejercicio de equilibrio. }\end{array}$ & $\begin{array}{l}30 \min \times 6 \\
\text { semanas } \times 5 \\
\text { sesiones }\end{array}$ & $\begin{array}{l}\uparrow \mathrm{E} \\
\uparrow \mathrm{MF} \\
\uparrow \mathrm{CV}\end{array}$ \\
\hline
\end{tabular}

Abreviaciones de la tabla: GC: grupo control; GE: grupo experimental; n: numero; $\uparrow \mathrm{E}$ : mejora en equilibrio; $\uparrow$ MF: aumento fuerza muscular; $\uparrow C V$ : mejora en la calidad de vida; $\uparrow$ MF: mayor movilidad funcional; $\uparrow A V D$ : mejora en las actividades de la vida diaria; $\uparrow$ PAR: mejora en la propiocepción de la articulación de la rodilla; $\uparrow E P$ : mayor estabilidad postural; $\uparrow \mathrm{MC}$ 


\section{RESULTADOS}

Entre los principales resultados de las intervenciones con Xbox Kinect se observó en el estudio de Karahan et al., (2015) un aumento en la escala de balance Berg pre intervención $(49.85 \pm 3.88)$ post intervención $(54.91 \pm 2.67)$ siendo estas diferencias estadísticamente significativas $\mathrm{p}<0.05$ también en los parámetros de calidad de vida, función social, percepción general de salud post-ejercicio en el grupo se observaron mejoras estadísticamente significativas $\mathrm{p}<0.05$. La evaluación post-ejercicio de la motivación de participación en el ejercicio del grupo de ejercicio de equilibrio doméstico con una escala Likert de 5 puntos reveló que el $7.1 \%(\mathrm{n}=3)$ de los participantes evaluó el estudio como "no placentero en absoluto", el 16.6\% $(\mathrm{n}=7)$ lo calificó como "desagradable", 42.8\% ( $\mathrm{n}=18)$ lo calificó como "moderadamente agradable ", 21.4\% ( $\mathrm{n}=9)$ lo calificó como "bastante agradable", y 11.9\% $(\mathrm{n}=5)$ lo calificó como "muy agradable". En el Grupo Xbox Kinect, 1 18.7\% $(\mathrm{n}=9)$ de los participantes evaluó el estudio como "moderadamente placentero", $37.5 \%(\mathrm{n}=18)$ lo consideró "bastante placentero", y 43.7\% $(\mathrm{n}=21)$ lo consideró "muy agradable". En el estudio de (Sadeghi et al., 2017) se observa una mejora en la propiocepción de rodilla en la pierna dominante en un ángulo de $30^{\circ}$ pre intervención $(6.0 \pm 1.1)$ post intervención $(3.5 \pm 1.1)$ en la pierna no dominante pre intervención $(6 \pm 1.1)$ post intervención $(3.8 \pm 0.8)$ en $45^{\circ}$ pierna dominante pre intervención $(5.1 \pm 1.6)$ post intervención $(3.1 \pm 0.9)$ pierna no dominante pre intervención $(5.9 \pm 1.6)$ post intervención $(3.1 \pm 0.9)$ en $60^{\circ}$ pierna dominante pre intervención $(7.2 \pm 1.5)$ post intervención $(3.0 \pm 0.6)$ pierna no dominante pre intervención $(7.9 \pm 1.4)$ y post intervención $(3.9 \pm 1.2)$ siendo estas diferencias estadísticamente significativas $\mathrm{p}<0,01$. Mientras que en el estudio de Bieryla (2015) se observaron mejoras estadísticamente significativas en la escala de balance Berg y la escala de balance avanzada de Fullerton $(\mathrm{p}<0.05)$. En el estudio de Shih, Wang, Cheng y Yang, (2016) se observó un aumento en la escala de balance Berg pre intervención $(50.9 \pm 5.32)$ post intervención $(53.2 \pm 2.86)$ en el control direccional pre intervención $(75.7 \pm 8.78)$ post intervención $(78.9 \pm 7.65)$ y disminución en la prueba Timed up and Go pre intervención $(9.5 \pm 2.45)$ post intervención $(8.71 \pm 1.8)$ siendo estas diferencias estadísticamente significativas $\mathrm{p}<0,05$ en adultos mayores con párkinson. En el estudio de Grigorova, et al., 2015 se observó una disminución en el tiempo de ejecución en la prueba Timed up and Go pre intervención $(19.1 \pm 1.2)$ post intervención (17.8 \pm 1.2$)$, un aumento en la escala de balance Berg pre intervención (35.2 \pm 1.9 ), post intervención $(46.8 \pm 2.6)$ y un aumento en el test Mini-Mental State Examination pre interven-

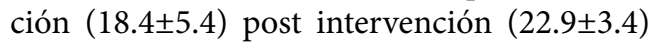
siendo estas diferencias estadísticamente significativas $\mathrm{p}<0,05$. Lee en el 2013 mostró un aumento en la fuerza muscular en flexión de hombro pre intervención $(4.14 \pm 1.95)$ post intervención $(5.57 \pm 2.70)$ extensión de hombro pre intervención $(3.85 \pm 2.04)$ post intervención $(5.57 \pm 2.37)$ extensión de codo pre intervención $(3.43 \pm 2.23)$ post intervención (5.71 \pm 2.93$)$ flexión de codo pre intervención $(3.86 \pm 2.27)$ post intervención $(6.29 \pm 2.81)$ y en FIM (medida de independencia funcional) pre intervención $(62.71 \pm 11.48)$ post intervención $(71.42 \pm 15.00)$ en pacientes con accidente cerebro vascular crónico. En el estudio de Lee et al., 2017 se observó un aumento en la escala de balance Berg pre intervención (43.35 \pm 6.23 ) post intervención $(46.19 \pm 5.57)$ y una disminución en el tiempo del test Timed up and Go pre intervención $(27.18 \pm 14.90)$ post intervención $(24.15 \pm 10.87)$ siendo estas diferencias estadísticamente significativas $\mathrm{p}<0,05$ en pacientes con accidente cerebro vascular. En el estudio de Kim et al., 2013 se observó un aumento en la fuerza de extensión de cadera pre intervención (20.70 \pm 5.77$)$ post intervención $(32.14 \pm 6.84)$ en la flexión de cadera $(22.69 \pm 12.04)$ post intervención (29.48 \pm 7.42$)$ aducción de cadera pre intervención $(26.51 \pm 7.03)$ post intervención (39.40 \pm 8.46$)$ abducción de cadera pre intervención $(23.80 \pm 7.73)$ post intervención (33.78 \pm 9.41$)$ siendo estas diferencias estadísticamente significativas $p<0,05$. En el estudio de Sato et al., 2015 se observó un aumento en la prueba de alcance funcional pre intervención $(29.36 \pm 4.40)$ post intervención $(33.88 \pm 3.94)$ y en la prueba de pararse y sentarse de la silla por 30 segundos pre intervención $(17.54 \pm 17.50)$ 
post intervención $(24.04 \pm 23.50)$ siendo estas diferencias estadísticamente significativas.

\section{DISCUSIÓN}

Las intervenciones mediante video juegos han mostrado mejoras en el equilibrio, movilidad funcional, fuerza muscular, propiocepción de la articulación de la rodilla, función cognitiva, los cuales poseen un impacto en el desempeño asociado al control motor, el que afecta directamente en la independencia funcional en este grupo etario, demostrando por tanto un efecto potencial en la calidad de vida de los adultos mayores. Esto se observó en intervenciones de 3, 6 y 8 semanas, mostrando una buena adherencia al juego con Xbox, siendo muy agradable en su utilización, y demostrando por lo tanto un efecto tanto en la adherencia como en la motivación de los usuarios en tratamientos funcionales. El efecto de los juegos digitales en el equilibrio se ha estudiado en personas con trastornos de movilidad (Darekar et al. 2015) y adultos mayores (Bisson et al., 2007; Szturm et al., 2011). Lo anterior se vuelve altamente relevante en función de que la motivación en estudios anteriores ha demostrado estar relacionada directamente con los resultados de rehabilitación (Gorsic, Cikajlo, \& Novak, 2017; Tuntland, et al., 2017).

Igualmente, otros estudios han demostrado que los video juegos muestran efectos positivos en parámetros físico-sociales, como habilidades cognitivas, coordinación, autoestima, autosuficiencia, atención, motivación y propiocepción en niños y adolescentes (Staiano \& Calvert, 2011a, 2011b), similar a los resultados observados en esta revisión. De igual manera las intervenciones mediante video juegos han mostrado mejoras sobre el equilibrio en adultos mayores, muy importante en prevención de caídas, funcionalidad y dependencia funcional.

Otro de los resultados observados en personas con accidente cerebro vascular crónico presentó una mejora en el equilibrio en la movilidad funcional y la fuerza muscular siendo ésta, una buena opción de intervención en este grupo de personas. En estudios ante- riores, la realidad virtual se ha utilizado en la rehabilitación de accidente cerebro vascular ya que puede mejorar el equilibrio a través de retroalimentación multisensorial y prácticas repetitivas (Pichierri et al., 2011). Este tipo de entrenamiento mediante realidad virtual combina la estimulación sensorial con la cognitiva (Saposnik et al., 2010). En una investigación realizada por Shin, Park \& Jang, 2015 se estudió la efectividad del entrenamiento de realidad virtual con la terapia ocupacional en el puntaje de SF-36 informando mejoras en su puntuación. En comparación con el entrenamiento convencional, el entrenamiento en realidad virtual proporciona un entorno más estimulante, enriquecedor y placentero que motiva a los pacientes a participar por completo en el programa, lo cual hace que la persona tenga una mayor adherencia a la intervención (Hung et al., 2014).

Otro de los resultados observados en este estudio, fue la mejora en la propiocepción de rodilla en distintos ángulos $\left(30^{\circ}, 45^{\circ}, 90^{\circ}\right)$, la propiocepción en la articulación de rodilla, es de importancia relevante para la estabilidad y realización de movimientos eficientes, pudiendo describir mejor como la información aferente derivada de los propioceptores en husos musculares, ligamentos y cápsulas, ayudan en el control motor, estabilidad articular y control postural (Daneshjoo, Mokhtar, Rahnama, \& Yusof, 2012). Siendo el control sensorio motor apropiado en las extremidades inferiores importante para el equilibrio y mantenimiento en la movilidad y estabilidad de la articulación (Sohn \& Kim., 2015). Déficits en el sistema propioceptivo, somato sensorial y vestibular ocurren con frecuencia en la vejez, siendo a menudo el resultado de trastornos de dolor músculo esquelético de inicio gradual, como daño traumático en ligamentos y músculos (Clark, Röijezon, \& Treleaven, 2015). Este deterioro conduce a una pérdida del control de la articulación de la rodilla, al equilibrio y, por ende, aumenta el riesgo de caída en este grupo etario (Relph \& Herrington, 2016). 


\section{CONCLUSIÓN}

A pesar de que los estudios difieren entre las intervenciones realizadas en cuanto al tiempo de duración de cada intervención, se observa que el tratamiento mediante ejercicio físico con consola Xbox Kinect proporciona mejoras en el equilibrio, fuerza muscular, movilidad funcional, función cognitiva, propiocepción en la articulación de rodilla, impactando directamente en la independencia funcional y calidad de vida en adultos mayores, favorece la motivación al tratamiento, y permite que al ser más agradable su práctica tiendan a una mayor adherencia en su ejecución. Lo anterior indica la importancia e impacto positivo que puede tener incorporarlo en Chile como tratamiento para la rehabilitación funcional en la práctica clínica en adultos mayores con alguna patología o sin patologías.

\section{REFERENCIAS BIBLIOGRÁFICAS}

Bieryla, K. (2015). Xbox Kinect training to improve clinical measures of balance in REFERENCIAS older adults: a pilot study. Aging Clinical and Experimental Research, 28(3), 451-457. doi:10.1007/ s40520-015-0452-y

Bisson, E., Contant, B., Sveistrup, H., Lajoie, Y. (2007). Functional balance and dualtask reaction times in older adults are improved by virtual reality and biofeedback training. Cyberpsychology \& Behavior, 10(1): 16-23. https://www.ncbi. nlm.nih.gov/pubmed/17305444

Burke, J.W, McNeill, M.D, Charles, D.K, Morrow, P.J, Crosbie, J.H, McDonough, S.M. (2009). Optimising engagement for stroke rehabilitation using serious games, The Visual Computer, 25(12), 1085-1099.

Casas, A., \& Izquierdo, M. (2012). Ejercicio físico como intervención eficaz en el anciano frágil. Anales del Sistema Sanitario de Navarra, 35, 69-85.
CASEN (2015). Adultos mayores. Síntesis de resultados. Recuperado de: http://observatorio.ministeriodesarrollosocial. gob.cl/casen-multidimensional/casen/ docs/CASEN 2015 Resultados adultos_mayores.pdf

Clark, N., Röijezon, U., \& Treleaven, J. (2015). Proprioception in musculoskeletal rehabilitation. Part 2: Clinical assessment and intervention. Manual Therapy, 20(3), 378-387. doi:https://doi. org/10.1016/j.math.2015.01.009

Choudhri, A., Siddiqui, A., Khan, N., \& Cohen, H. (2015). Understanding bibliometric parameters and analysis. Radiographics : a review publication of the Radiological Society of North America. Inc., 35(3), 736-746.

Corazza, S., Laux, R., Foesch, M., Santos, D., Machado, R., Macedo, T., Piovesan, A., \& Mezzomo, S. (2016). Benefícios Do Treinamento Funcional Para O Equilíbrio E Propriocepção De Deficientes Visuais. Revista Brasileira de Medicina do Esporte, 22(6), 471475. https://dx.doi.org/10.1590/1517869220162206164644

Darekar, A., McFadyen, B.J., Lamontagne, A., Fung, J. (2015). Efficacy of virtual reality-based intervention on balance and mobility disorders post-stroke: a scoping review. Journal of NeuroEngineering and Rehabilitation 12:46.

Daneshjoo, A., Mokhtar, A., Rahnama, N., \& Yusof, A. (2012). The Effects of Comprehensive Warm-Up Programs on Proprioception, Static and Dynamic Balance on Male Soccer Players. PLoS ONE, 7(12), e51568. doi:10.1371/journal.pone.0051568

Galna, B., Barry, G., Jackson, D., Mhiripiri, D., Olivier, P., Rochester, L. (2014). Accuracy of the Microsoft Kinect sensor for measuring movement in people with Parkinson's disease. Gait Posture, 39(4):1062-8. https://www.ncbi.nlm. nih.gov/pubmed/24560691 
Grigorova, K., Dimitrova, A., Lubenova, D., Zaharieva, D., Vasileva, D. (2015). Feasibility of interactive video games for influence on balance in institutionalized elderly people. Journal of Physical Education and Sport, 15(31), 429 - 432.

Gómez, A., Rodríguez, V., Maldonado, V., Casajús, A., \& Ara, I. (2012). Envejecimiento y composición corporal: la obesidad sarcopenica en España. Nutrición Hospitalaria, 27(1), 22-30.

Gorsic, M., Cikajlo, I., \& Novak, D. (2017). Competitive and cooperative arm rehabilitation games played by a patient and unimpaired person: effects on motivation an exercise intensity. Journal of Neuroengineering and rehabilitation, 14(23). doi: 10.1186/ s12984-017-0231-4

Hernández, J., \& Ramírez, R. (2017). Efectos del entrenamiento vibratorio sobre el riesgo de caída en adultos mayores institucionalizados: una revisión breve. Revista Ciencias de la Actividad Física UCM, 18 (2), 1-7.

Hung, JW., Chou, CX., Hsieh, YW., Wu, WC., Yu, MY., Chen, PC., Chang, HF., Ding, SE. (2014). Randomized comparison trial of balance training by using exergaming and conventional weight-shift therapy in patients with chronic stroke. Arch Phys Med Rehabil, 95(9):16291637. https://www.ncbi.nlm.nih.gov/ pubmed/24862764

Jorgensen, M., Laessoe, U., Hendriksen, C., Nielsen, O., \& Aagaard, P. (2013). Efficacy of Nintendo Wii training on mechanical leg muscle function and postural balance in community-dwelling older adults: a randomized controlled trial. The Journals of Gerontology: Series $A, 68(7), 845-852$. doi:10.1093/gerona/ gls222
Karahan, A., Tok, F., Taskin, H., Kucuksarac, S., Basaran, A., \& Yildirim, P. (2015). Effects of Exergames on Balance, Functional Mobility, and Quality of Life of Geriatrics Versus Home Exercise Programme: Randomized Controlled Study. Cent Eur J Public Health. Suppl:S14-8. https://www.ncbi.nlm. nih.gov/pubmed/26849537

Kim, J., Son, J., Ko, N., \& Yoon, B. (2013). Unsupervised Virtual Reality-Based Exercise Program Improves Hip Muscle Strength and Balance Control in Older Adults: A Pilot Study. Arch Phys Med Rehabil, 94(5):937-43.

Kim, T., Xiong, S. (2016). Comparison of seven fall risk assessment tools in community-dwelling Korean older women. Ergonomics 60(3):421-429. doi: $\underline{10.1080 / 00140139.2016 .1176256}$

Ko, C., Chang, Y., Jeong, B., Kang, S., Ryu, J., \& Kim, G. (2017). Effect of knee sleeves on coordination of lower-limb segments in healthy adults during level walking and one-leg hopping. PeerJ, 5:e3340. doi: $10.7717 /$ peerj.3340

Lee, HC., Huang, CL., Ho, SH., Sung, WH. (2017). The Effect of a Virtual Reality Game Intervention on Balance for Patients with Stroke: A Randomized Controlled Trial. Games Health J. 6(5): 303311. doi: $10.1089 / \mathrm{g} 4 \mathrm{~h} .2016 .0109$

Lee, G. (2013). Effects of training using video games on the muscle strength, muscle tone, and activities of daily living of chronic stroke patients. J. Phys. Ther. Sci. 25(5): 595-597. doi: 10.1589/ jpts. 25.595 
Lima, F., Camillo, C., Gobbo, L., Trevisan, I., Nascimento, W., Silva, B., Lima, M., Ramos, D., Ramos, E. (2018). Resistance Training using Low Cost Elastic Tubing is Equally Effective to Conventional Weight Machines in Middle-Aged to Older Healthy Adults: A Quasi-Randomized Controlled Clinical Trial. Journal of Sports Science \& Medicine, 17(1), 153-160.

Lopes, P., Pereira, G., Lodovico, A., Bento, P., \& Rodacki, A. (2016). Strength and Power Training Effects on Lower Limb Force, Functional Capacity, and Static and Dynamic Balance in Older Female Adults. Rejuvenation Research, 19(5), 385-393. doi:10.1089/rej.2015.1764

Masud, T., \& Morris, R. (2001). Epidemiology of falls. Age and Ageing, 30-S4: 3-7.

Nelson, M., Rejeski, WJ., Blair, SN., Duncan, PW., Judge, JO., King, AC., Macera, CA., Castaneda, SC. (2007). Physical Activity and Public Health in older Adults: Recommendations from the American college of Sports Medicine and the American Heart Association. Medicine Science in Sports and Exercise; 39(8): 1435-1445

OMS. (2018). Organizacion Mundial de la Salud. Envejecimiento y ciclo de vida. Recuperado de: http://www.who.int/ ageing/about/facts/es/

Padala, K., Padala, P., Lensing, S., Dennis, R., Bopp, M., Parkes, C., . . . Sullivan, D. (2017). Efficacy of Wii-Fit on Static and Dynamic Balance in Community Dwelling Older Veterans: A Randomized Controlled Pilot Trial. Journal of Aging Research, 2017, 4653635. doi:10.1155/2017/4653635

Petronila, L., Aragón, S., \& Calvo, B. (2017). Caídas en ancianos institucionalizados: valoración del riesgo, factores relacionados y descripción. Gerokomos, 28, 2-8.
Pichierri G, Wolf P, Murer K, de Bruin ED. (2011). Cognitive and cognitive-motor interventions affecting physical functioning: a systematic review. $B M C$ Geriatrics, 11:29. doi: 10.1186/14712318-11-29

Relph, N., \& Herrington, L. (2016). The effects of knee direction, physical activity and age on knee joint position sense. The Knee, 23(3), 393-398. doi:10.1016/j. knee.2016.02.018

Sadeghi, H., Hakim, M., Hamid, T., Amri, S., Razeghi, M., Farazdaghi, M., \& Shakoor, E. (2017). The effect of exergaming on knee proprioception in older men: A randomized controlled trial. Archives of Gerontology and Geriatrics, 69: 144-150. doi:https://doi. org/10.1016/j.archger.2016.11.009

Saposnik, G., Mamdani, M., Bayley, M., Thorpe, KE., Hall, J., Cohen, LG., Teasell, R; EVREST Steering Committee; EVREST Study Group for the Stroke Outcome Research Canada Working Group. (2010). Effectiveness of Virtual Reality Exercises in STroke Rehabilitation (EVREST): rationale, design, and protocol of a pilot randomized clinical trial assessing the Wii gaming system. Int J Stroke, 5(1): 47-51.

Sato, K., Kuroki, K., Saiki, S., Nagatomi, R., 2015. Improving walking, muscle strength, and balance in the elderly with an exergame using Kinect: a randomized controlled trial. Games Health J. 4(3), 161-167.

SENAMA. (2011). Servicio Nacional de adulto mayor. Estudio Nacional de la dependencia en personas mayores. Recuperado de: www.senama.cl/filesapp/cuentapublica2011.

Shin J-H, Park SB, Jang SH. (2015). Effects of game-based virtual reality on healthrelated quality of life in chronic stroke patients: A randomized, controlled study. Comput Biol Med, 63:92-98. 
Shih, M., Wang, R., Cheng, S., \& Yang, Y. (2016). Effects of a balance-based exergaming intervention using the Kinect sensor on posture stability in individuals with Parkinson's disease: a single-blinded randomized controlled trial. Journal of NeuroEngineering and Rehabilitation, 13(1), 78. doi:10.1186/ s12984-016-0185-y

Shimada, H., Obuchi, S., Furuna, T., Suzuki, T. (2004). New intervention program for preventing falls among frail elderly people:the effects of perturbed walking exercise using a bilateral separated treadmill. Am J Phys Med Rehabil, 83(7):493-9.

Sin H, Lee G. (2013). Additional virtual reality training using Xbox kinect in stroke survivors with hemiplegia. Am J Phys Med Rehabil; 92:871-880.

Silsupadol, P., Siu, KC., Shumway, A.,Woollacott, MH. (2006). Training of balance under single-and dual-task conditions on older adults whit balance impaierment. Phys Ther; 86(2):269-81

Smith, S., \& Schoene, D. (2012). The use of exercise-based videogames for training and rehabilitation of physical function in older adults: current practice and guidelines for future research. Aging Health, 8(3), 243-252.

Sohn, J., \& Kim., S. (2015). Falls study: Proprioception, postural stability, and slips. Biomed Mater Eng, 26, 693-703.

Staiano, A., \& Calvert, S. (2011a). Exergames for Physical Education Courses: Physical, Social, and Cognitive Benefits. Child development perspectives, 5(2), 93-98. doi:10.1111/j.1750$\underline{8606.2011 .00162 . \mathrm{x}}$
Staiano, A., \& Calvert, S. (2011b). The promise of exergames as tools to measure physical health. Entertainment computing, 2(1), 17-21. doi:10.1016/j. entcom.2011.03.008

Szturm T, Betker A L, Moussavi Z, Desai A, Goodman V, (2011) Effects of an Interactive Computer Game Exercise Regimen on Balance Impairment in Frail Community-Dwelling Older Adults: A Randomized Controlled Trial, Phys Ther; 91:1449-1462

Tuntland, H., Kjeken, I., Langeland, E., Folkestad, B., Espehaug, B., Forland, O., \& Aaslund, M. (2017) Predictors of outcomes following reablement in community-dwelling older adults. Clinical Interventions in Aging. 12, 55-67. doi: $10.2147 / C I A . S 125762$

Vernon S, Paterson K, Bower K, McGinley J, Miller K, Pua YH, et al.(2015). Quantifying individual components of the timed up and go using the kinect in people living with stroke. Neurorehab Neural Repair. 29:48-53.

\section{Dirección para correspondencia}

María Francisca Rauch Gajardo

Terapeuta Ocupacional,

Magíster en Neurociencias de la Educación.

Académico Escuela de Terapia Ocupacional,

Facultad de Salud,

Universidad Santo Tomás, Talca, Chile

Contacto:

mrauch@santotomas.cl

Recibido: 06/06/2018

Aceptado: 28/06/2018 